\title{
TERAPIAS ALTERNATIVAS E COMPLEMENTARES UTILIZADAS POR ENFERMEIRAS OBSTETRAS EM UM CENTRO DE PARTO NORMAL
}

\section{ALTERNATIVE AND COMPLEMENTARY THERAPIES USED BY NURSES, OBSTETRICIANS IN A NORMAL DELIVERY CENTER}

\section{TERAPIAS ALTERNATIVAS Y COMPLEMENTARIAS UTILIZADAS POR LAS ENFERMERAS, OBSTETRAS EN UN CENTRO DE PARTO NORMAL}

\author{
Inglid Gleici Ferreira Alves ${ }^{1}$, Maria Betânia Dantas Matias², Juliana Manoela Santos Freitas ${ }^{3}$, \\ Rosa Áurea Quintella Fernandes ${ }^{4}$
}

\section{RESUMO}

Introdução: As Terapias Alternativas e Complementares são métodos não farmacológicos para o alívio da dor e evolução do trabalho de parto. Essas terapias permitem não só que a parturiente exerça sua autonomia durante o trabalho de parto, mas também que vivencie este momento de forma menos traumática. Entre as terapias não farmacológicas estão o banho de aspersão, a massagem, a deambulação, o balanço pélvico, a bola suíça, o agachamento e a hidromassagem. Objetivo: verificar o uso de Terapias Alternativas e Complementares por enfermeiras obstetras em um Centro de Parto Normal. Método: estudo exploratório, descritivo, retrospectivo, documental com abordagem quantitativa, desenvolvido com base em banco de dados de pesquisa primária. A amostra foi constituída por 300 prontuários. O estudo primário foi aprovado por Comitê de Ética em Pesquisa. Resultados: as terapias não farmacológicas mais utilizadas pelas enfermeiras obstetras na assistência às mulheres durante o trabalho de parto de risco habitual foram: deambulação $(58,7 \%)$, banho de aspersão $(51,7 \%)$ e balanço pélvico $(42,7 \%)$. A bola suíça, o agachamento e a massagem foram empregadas em percentuais menores (14,0\%; 11,3\%; 10,7\%). Conclusão: As enfermeiras obstetras do Centro de Parto Normal analisado tem utilizado algumas terapias não farmacológicas com maior intensidade que outras. Recomenda-se que mais estudos sejam realizados para avaliar se outros Centros de Parto têm sido mais efetivos na utilização dessa tecnologia.

DESCRITORES: Parto humanizado; Dor do parto; Trabalho de parto; Terapias complementares.

\begin{abstract}
Introduction: Alternative and complementary therapies are non-pharmacological methods for the relief of pain and evolution of labor. These therapies not only allow parturient to exercise their autonomy during labor, but also to experience this moment in a less traumatic way. Among the non-pharmacological therapies are the sprinkling bath, the massage, the wandering, pelvic balance, Swiss ball, squatting and hydromassage.Objective: To check the use of alternative and complementary therapies by obstetrician nurses in a Normal Labor Center. Method: Exploratory study, descriptive, retrospective, documentary with quantitative approach, developed based on primary research database. The sample was made up of 300 charts. The primary study was approved by the Research Ethics Committee. Results: The nonpharmacological therapies most used by obstetrician nurses in assisting women during the usual risk childbirth work were walking (58.7\%), sprinkling bath (51.7\%) and pelvic balance (42.7\%). The Swiss ball, squat and massage were employed in smaller percentages (14.0\%; 11.3\%; 10.7\%). Conclusion: The obstetrician nurses at the Normal Labor Center analyzed have used some non-pharmacological therapies with more intensity than others. It is recommended that more studies be carried out to assess whether other birth centres have been more effective in using this technology. DESCRIPTORS: Humanized childbirth; Pain of childbirth; Labor; Complementary therapies.
\end{abstract}

\footnotetext{
Acadêmica de Enfermagem da Universidade UNIVERITAS/UNG - Guarulhos - SP.

Acadêmica de Enfermagem da Universidade UNIVERITAS/UNG - Guarulhos - SP.

Enfermeira Obstetra, Mestre em Ciências pela Universidade UNIVERITAS/UNG - SP.

4 Obstetriz, Doutora em Enfermagem, Docente do Programa de Pós-Graduação Mestrado/Doutorado da Universidade UNIVERITAS/UNG Guarulhos - SP.
} 


\section{RESUMEN}

Introducción: Las Terapias Alternativas y Complementarias son métodos no farmacológicos para el alivio del dolor y evolución del trabajo de parto. Esas terapias permiten no sólo que la parturiente ejerza su autonomía durante el trabajo de parto, pero también que vivencie este momento de manera menos traumática. Entre las terapias no farmacológicas están el baño de aspersión, el masaje, la deambulación, el balance pélvico, la bola suiza, el agachamiento y el hidromasaje. Objetivo: verificar el uso de Terapias Alternativas y Complementarias por enfermeras obstetras en un Centro de Parto Normal. Método: estudio exploratorio, descriptivo, retrospectivo, documental con abordaje cuantitativo, desarrollado sobre la base de datos de investigación primaria. La muestra fue constituida por 300 prontuarios. El estudio primario fue aprobado por Comité de Ética en Investigación. Resultados: las terapias no farmacológicas más utilizadas por las enfermeras obstetras en la asistencia a las mujeres durante el trabajo de parto de riesgo habitual fueron: deambulación (58,7\%), baño de aspersión (51,7\%) y balance pélvico (42,7\%). La pelota suiza, el agachamiento y el masaje fueron empleadas en porcentajes menores (14,0\%, 11,3\%, 10,7\%). Conclusión: Las enfermeras obstetras del Centro de Parto Normal analizado han utilizado algunas terapias no farmacológicas con mayor intensidad que otras. Se recomienda realizar más estudios para evaluar si otros Centros de Parto han sido más efectivos en la utilización de esta tecnología.

DESCRIPTORES: Parto humanizado; Dolor del parto; Trabajo de parto; Terapias complementarias;

\section{INTRODUÇÃO}

A assistência obstétrica humanizada exige do profissional de saúde conhecimento, prática e atitudes que favoreçam o acolhimento à mulher e seu companheiro no serviço de saúde, desde o planejamento familiar, pré-natal, trabalho de parto e parto. A equipe de saúde, como um todo, deve proporcionar o bem-estar tanto da mulher quanto do bebê, evitando intervenções desnecessárias e permitindo sua privacidade, participação e autonomia em todas as etapas do trabalho de parto e parto'1.

Um dos medos mais frequentes das parturientes está relacionada à dor que enfrentarão durante o trabalho de parto, sendo amplamente variável e sujeita à influência de vários aspectos, psíquicos, culturais, familiares, medo ou relato de experiências anteriores ${ }^{3}$. A dor é um fenômeno complexo, subjetivo, de causa multifatorial que cada pessoa sente e descreve de maneira diferente ${ }^{2}$, portanto, cada mulher vivencia a dor no parto de forma muito pessoal.

Um dos objetivos da assistência ao parto de forma humanizada é proporcionar à parturiente a tolerância e o autocontrole da dor, causadas pela contração uterina durante o trabalho de parto ${ }^{2}$.

O enfermeiro obstetra/obstetriz deve criar situações que estabeleçam um vínculo de confiança com a parturiente, permitindo que a mulher seja protagonista do seu momento, que é único. A parturiente deve ser ouvida em todas as fases do trabalho de parto, e um ambiente tranquilo e terapêutico deve ser criado, de modo a favorecer à parturiente total liberdade e participação ativa na escolha e no uso de terapias alternativas no alívio da dor. As terapias alternativas utilizadas de forma adequada, respeitando os limites de cada mulher permitem que cada uma vivencie seu trabalho de parto de forma menos traumática ${ }^{4}$.

Para controlar a dor e outros sintomas durante o trabalho de parto, vem sendo utilizadas terapias farmacológicas e não-farmacológicas, porém as medidas farmacológicas, mesmo sendo efetivas para a diminuição ou inibição da dor podem atrapalhar o processo fisiológico do parto ${ }^{5}$.

A utilização de Terapias Alternativas e Complementares (TACs) estão se tornando cada vez mais frequentes, embora mais estudos controlados sejam necessários para consolidar sua efetividade no controle da dor. Essas terapias são vantajosas, seja pelo custo-benefício, seja pela autonomia que é dada ao profissional enfermeiro ${ }^{5}$

"Não há uma definição unanime em relação ao conceito de TAC que podem ter outras denominações como: medicina complementar, terapias complementares, medicina alternativa entre outros. A Biblioteca Nacional de Medicina (Nathional Library of Medicine) incluiu o descritor (Medical Subject Headings-MeSH) Complementary Therapies e o definiu como sendo práticas terapêuticas que não são consideradas como parte integrante da prática médica convencional alopática"5.

O Ministério da Saúde ${ }^{1}$ recomenda que se utilize na assistência ao parto as práticas demonstradamente úteis 
(Boas Práticas), nas quais a aplicação de Métodos não invasivos e não farmacológicos de alívio da dor, como massagens e técnicas de relaxamento está incluída.

Dentre as TACS's as mais citadas são: a aromaterapia, massagem, banho quente de aspersão, bola suíça, deambulação e mudança de posição, musicoterapia, acupuntura, estimulação elétrica transcutânea e crioterapia ${ }^{9}$.

\section{- $\quad$ Aromaterapia}

A aromaterapia utiliza a essência de algumas plantas para o alívio da dor, e estimula a liberação de substâncias no corpo, substâncias essas que tem poder relaxante, estimulante e sedativo ${ }^{6}$. A aromaterapia contribui para aliviar o desconforto do trabalho de parto e pelo fato do sistema límbico ter ligações com o bulbo olfatório a utilização desta prática auxilia a mãe na parte emocional ${ }^{7}$.

\section{- Massagem}

A massagem ativa a estimulação sensorial, e por esse motivo este método auxilia no alívio da dor, propicia o relaxamento e diminui o estresse causado pelo trabalho de parto ${ }^{8}$.Essa TAC é uma das mais simples e de baixo custo, sendo também altamente eficaz quando associado com outros métodos não farmacológicos, como a deambulação, melhorando assim o fluxo sanguíneo e a oxigenação de todos os tecidos ${ }^{8,9}$.

A massagem pode ser aplicada pelo acompanhante ou até mesmo pela própria parturiente. Existem vários locais do corpo para sua aplicação como cabeça, abdome, ombros entre outros, dependendo do local de desconforto da parturiente. O local mais comum para a aplicação da massagem é a região lombar, por ser a mais dolorida durante as contrações ${ }^{8,9}$.

\section{- Banho quente de aspersão}

O banho quente de aspersão faz com que um calor superficial seja estimulado, e dependendo do tempo de aplicação, promove um efeito local ou até mesmo geral, pois provoca o relaxamento muscular induzido pela vasodilatação periférica causada pela água aquecida que é utilizada ${ }^{3,9}$. Há diminuição da liberação de catecolaminas e o aumento de endorfina o que propicia o alívio da dor, e consequentemente reduz a tensão e a ansiedade demonstrada pela parturiente ${ }^{9}$.

\section{- Bola suíça}

A bola suíça é utilizada na fisioterapia para tratamento neurológico e outros tipos de terapias. O balanço pélvico que a bola produz durante o trabalho de parto, trabalha vários músculos, principalmente o do assoalho pélvico e o pubo coccígeo, auxiliando na descida e rotação do feto, além de tornar as contrações mais eficazes e ajudar no processo de dilatação. Esse método é uma forma de tornar a parturiente independente e ativa durante o trabalho de parto e nascimento ${ }^{3}$.

\section{- Deambulação e Mudança de posição}

Uma terapia alternativa simples e fácil de ser aplicada durante o trabalho de parto, pois não atribui custo a instituição. A sua utilização proporciona à parturiente a liberdade para a deambulação e mudança de posição no decorrer do trabalho de parto, favorecendo a diminuição da dor e o tempo do trabalho de parto. Consequentemente melhora a contratilidade uterina, permitindo mais conforto, viabilizando a participação e entrosamento da parturiente e acompanhante, tornando o trabalho de parto um momento família ${ }^{1,9,10}$.

Estudos mostram que as mudanças de posição a cada 30 minutos trazem excelentes resultados sobre a atividade e efetividade uterina, além de permitir que a parturiente descubra e escolha a posição a qual ofereça melhor conforto ${ }^{9,10}$.

\section{- Musicoterapia}

Ciência do século $X X$ que estuda a influência da música com fins terapêuticos nos seres humanos ${ }^{11}$.A música tem um papel essencial no processo da assistência humanizada, considerada uma ferramenta complementar na promoção de conforto e relaxamento. Possibilita a criação de um ambiente acolhedor, garantindo sua efetividade no manejo da dor, ansiedade e estresse emocional e pode ser associada a outras TACs ${ }^{9,11}$.

\section{- Estimulação Elétrica Transcutânea}

Método não farmacológico no alívio da dor que contribui para a diminuição do uso de fármacos analgésicos e anestésicos, estimulando a prática do parto humanizado ${ }^{9}$.A estimulação elétrica transcutânea contribui para a diminuição da dor pelos estímulos elétricos. A técnica prevê o uso de eletrodos superficiais em áreas pré-estabelecidas T10 à L1 e nas vértebras S2 e S41.

\section{- Crioterapia}

Consiste na aplicação de compressas de gelo na região lombar, utilizando cinta específica por período de 20 minutos, a efetividade desta terapia se dá na fase ativa do período de dilatação, propiciando o alívio da dor 
sem prejudicar a dinâmica uterina. Este alívio da dor decorre da aplicação do gelo local, pois o mesmo estimula a liberação de endorfinas ${ }^{9,12 .}$

O enfermeiro obstetra/obstetriz deve conhecer a importância das TACs e seu manejo como medidas não-farmacológicas para o alivio da dor e aplica-las em sua prática assistencial de modo a tornar o trabalho de parto e parto, um momento menos tenso na vida da mulher $^{5}$ Além disso, traz o reconhecimento do profissional enfermeiro, pois sua assistência torna-se mais humanizada.

As TACs começaram a ser mais aplicadas a partir da criação dos Centros de Parto Normal (CPN) que privilegia a assistência ao parto de risco habitual realizado por enfermeira obstetra/obstetriz. Entretanto, sua prática ainda não está completamente incorporada à rotina de assistência ao parto em todas as instituições de saúde.

Conhecer o uso das TACs por enfermeiras/obstetrizes que atuam em CPN é importante para incentivar e disseminar sua utilização na assistência à mulher em trabalho de parto para maior conforto da parturiente.

Esse estudo teve como objetivo, verificar o uso das Terapias Alternativas e Complementares por enfermeiras obstetras/obstetrizes em um Centro de Parto Normal.

\section{MÉTODO}

Trata-se de estudo exploratório, descritivo, retrospectivo, documental com abordagem quantitativa. $\mathrm{O}$ estudo foi desenvolvido com base em banco de dados de uma pesquisa primária. O estudo primário foi realizado em CPN intra-hospitalar de uma instituição pública, de grande porte que atende pacientes do Sistema Único de Saúde (SUS) localizado no Estado de São Paulo. O CPN atende gestantes de risco habitual e a assistência é de responsabilidade da enfermeira obstetra.

A população do estudo primário foi constituída por todos os prontuários das mulheres que tiveram seus partos no CPN, no período de janeiro a dezembro de 2015. Para o cálculo amostral foi utilizado o programa estatístico STATS 2.0. Considerou-se o total de 1221 partos realizados no CPN no ano de 2015, porcentagem máxima aceitável de erro de $5 \%$, nível de percentagem estimada de $50 \%$ e nível de confiança de $95 \%$. A amostra representativa do total de partos foi de 300 prontuários.

A amostragem foi aleatória simples e o sorteio dos prontuários foi realizado no programa Randomized.com.
Critérios de inclusão para que o prontuário integrasse a amostra: ser sorteado; que o parto tivesse sido realizado por enfermeira obstetra/ obstetriz; prontuário disponível no arquivo no momento da coleta dos dados. Foram excluídos os prontuários das mulheres que deram à luz no CPN mas, atendidas na assistência ao parto pela pesquisadora do estudo primário. No estudo atual foram selecionadas como variáveis de interesse: dados sociodemográficos e obstétricos e as terapias alternativas utilizadas na assistência às parturientes.

O projeto primário foi aprovado por Comitê de Ética em Pesquisa parecer número 1.429.319, o estudo atual não necessitou nova provação.

Os dados de interesse para esta pesquisa foram coletados do banco de dados da pesquisa primária e inseridos em uma planilha Excel® versão 2013. Os dados foram submetidos a análise descritiva e as variáveis quantitativas apresentadas em números absolutos, frequência e média.

\section{RESULTADOS}

Nesse estudo foram analisados 300 prontuários de mulheres que deram à luz em um Centro de Parto Normal intra-hospitalar. Os resultados apontam que eram mulheres jovens com mediana de 22 anos de idade com intervalo interquartil (19-28).

Tabela 1. Características sociodemográficas das puérperas que compuseram a amostra. São Paulo, 2017.

\begin{tabular}{lrc} 
Variáveis* $^{*}$ & $\mathbf{N}$ & $\%$ \\
\hline Situação conjugal $(\mathrm{n}=297)$ & & \\
Com companheiro & 92 & 30,9 \\
Sem companheiro & 205 & 69,1 \\
Atividade remunerada $(\mathrm{n}=286)$ & & \\
Não & 171 & 59,8 \\
Sim & 115 & 40,2 \\
Escolaridade em anos $(\mathrm{n}=299)$ & & \\
1-4 & 19 & 6,4 \\
5-8 & 110 & 36,8 \\
9-11 & 163 & 54,5 \\
$\geq 12$ & 7 & 2,3 \\
\hline
\end{tabular}

* foram computados os prontuários no qual contavam a informação

Observa-se na Tabela 1 que a maioria das mulheres não tinham companheiro $(69,1 \%)$, não exercia atividade remunerada $(59,8 \%)$ e tinham entre 9 e 11 anos de estudo $(54,5 \%)$. 
Tabela 2. Dados obstétricos das parturientes que integraram a amostra. São Paulo, 2017.

\begin{tabular}{lcc}
\hline Variáveis $\mathbf{( n = 3 0 0 )}$ & $\mathbf{N}$ & $\%$ \\
\hline Paridade & & \\
Nenhuma & 133 & 44,3 \\
1 a 2 & 135 & 45,0 \\
$\geq 3$ & 32 & 10,7 \\
Número de consultas no pré-natal & & \\
0 & 8 & 2,7 \\
1-3 & 29 & 9,7 \\
$4-6$ & 72 & 24 \\
$\geq 7$ & 191 & 63,6 \\
\hline
\end{tabular}

Na Tabela 2, observa-se que $44,3 \%$ eram primigestas e $45,0 \%$ tinham 1 ou 2 filhos. Das 300 puérperas incluídas na pesquisa, pelo menos $63,6 \%$ compareceram a 7 ou mais consultas no pré-natal, entretanto $12,4 \%$ delas realizaram número de acompanhamento inferior ao preconizado pelo Ministério da Saúde (6 consultas) $)^{13}$.

Tabela3. Frequência das TerapiasAlternativas Complementares utilizadas por enfermeiras obstetras em CPN. São Paulo 2017.

\begin{tabular}{lcc}
\hline $\begin{array}{l}\text { Terapias Alternativas } \\
\text { Complementares }(\mathbf{n}=\mathbf{3 0 0})\end{array}$ & $\mathbf{N}$ & $\%$ \\
\hline Deambulação & 124 & 41,3 \\
Não & 176 & 58,7 \\
Sim & & \\
Banho de aspersão & 145 & 48,3 \\
Não & 155 & 51,7 \\
Sim & & \\
Balanço Pélvico & 172 & 57,3 \\
Não & 128 & 42,7 \\
Sim & & \\
Bola & 258 & 86,0 \\
Não & 42 & 14,0 \\
Sim & & \\
Agachamento & 266 & 88,7 \\
Não & 34 & 11,3 \\
Sim & & \\
Massagem & 268 & 89,3 \\
Não & 32 & 10,7 \\
Sim & & \\
Hidromassagem & 275 & 91,7 \\
Não & 25 & 8,3 \\
Sim & & \\
\hline
\end{tabular}

$\mathrm{Na}$ Tabela 3, observa-se que as terapias não farmacológicas mais utilizadas na assistência às parturientes foram: deambulação $(58,7 \%)$, banho $(51,7 \%)$ e balanço pélvico $(42,7 \%)$. A terapia menos utilizada foi a hidromassagem $(8,3 \%)$.

\section{DISCUSSÃO}

Nesse estudo predominaram as mulheres jovens, com mediana de 22 anos de idade, sem companheiro, sem trabalho remunerado e com escolaridade entre 9-11 anos de estudo.

Estudos ${ }^{14,15}$ que avaliaram a utilização das terapias alternativas em parturientes identificaram tratar-se, também, de mulheres jovens, média de idade de 21,5 anos e faixa etária entre 20 e 35 anos, respectivamente.

Os estudos ${ }^{14,15}$ mencionados encontraram resultados diferentes no que tange à situação conjugal e nível de escolaridade, uma vez que as mulheres eram em sua maioria casadas e tinham escolaridade menor do que a identificada no estudo atual. Quanto ao trabalho remunerado os dados se assemelham porque a maioria não exercia atividade remunerada.

As terapias não farmacológicas mais utilizadas pelas enfermeiras obstetras na assistência às mulheres durante o trabalho de parto, neste estudo, foram a deambulação (58,7\%), o banho de aspersão $(51,7 \%)$ e o balanço pélvico $(42,7 \%)$. Esses resultados diferem de estudo sobre as terapias alternativas no trabalho parto ${ }^{5}$ que identificou percentual de deambulação de $68,2 \%$ e de banho de chuveiro $65,8 \%$. O mesmo estudo aponta que a massagem foi utilizada em $51,2 \%$ das parturientes enquanto no estudo atual esse percentual foi de apenas $10,7 \%$, e não houve o uso do balanço pélvico.

O banho de aspersão e imersão foram as terapias alternativas mais identificadas (92,9\%) em estudo que avaliou a assistência em Centro de Parto Normal, a deambulação obteve $47,6 \%$ e a massagem foi a menos utilizada $(29,8 \%)^{16}$. Em pesquisa realizada em Centro de Parto extra hospitalar as terapias de alívio da dor mais empregadas foram: o banho de aspersão (84\%), a deambulação $(68 \%)$, a massagem $(60,1 \%)$ e o exercício com a bola suíça $(51,7 \%)^{17}$.

Estudo que analisou as boas práticas na assistência ao parto, identificou que $74 \%$ das enfermeiras obstetras utilizaram os métodos não farmacológicos para dor ${ }^{18}$.

Os métodos não farmacológicos na atenção ao trabalho de parto, entre eles a deambulação, são indicados 
por representarem menor risco para a mulher e se mostrarem efetivos no alívio da dor ${ }^{19}$.

A deambulação é recomendada nas primeiras três horas da fase ativa do trabalho de parto porque facilita a descida e a apresentação fetal ${ }^{20,21}$ trazendo benefícios tanto para a mãe quanto para bebê, diminuindo o tempo de trabalho de parto e os escores de dor.

O banho de chuveiro é outra terapia não farmacológica indicada para o alívio da dor. Estudo ${ }^{14}$ que avaliou algumas terapias não farmacológicas, apontou diferença estatisticamente significativa no alívio da dor com o uso do banho de chuveiro.

Os estudos têm apontado a deambulação, o banho de imersão e a massagem como as terapias complementares mais utilizada, pelas enfermeiras obstetras ${ }^{22}$.

O emprego das terapias não farmacológicas na atenção ao trabalho de parto além de serem de fácil aplicabilidade, reduzem a medicalização do parto, permitem a participação ativa do acompanhante durante esse processo e estão alinhadas à política de humanização do parto ${ }^{23}$.

Revisão de estudos randomizados sobre o uso das terapias não farmacológicas de alívio da dor no trabaIho de parto, analisou o banho de imersão, a massagem e a aromaterapia e concluiu que cada método tem sua importância, mas é necessário definir em que fase do período de trabalho de parto o método deve ser implementado ${ }^{24}$

Estudo $^{4}$ de revisão integrativa aponta as terapias complementares como recurso importante no alívio da dor no trabalho de parto e no maior conforto da mulher durante esse processo.

Assim, pergunta-se: por que esses recursos não têm sido integralmente utilizados na assistência ao parto?

Alguns estudos ${ }^{18,5,21}$ referem que possivelmente essas terapias não são utilizadas pelos enfermeiros por influência de fatores como: valorização da assistência baseada no modelo biomédico, a falta de especialização das enfermeiras em obstetrícia, falta de conhecimento em relação ao respaldo legal para essa prática e falta de orientação e indicação dos profissionais às parturientes $\mathrm{O}$ que sugere a necessidade de educação permanente, sobre a importância dessas terapias na assistência ao trabalho de parto, para os enfermeiros das unidades básicas de saúde até as unidades hospitalares ${ }^{21}$.

Há que se considerar na análise dos resultados do estudo atual, se as terapias não foram utilizadas com a frequência esperada por falta de interesse da enfermeira ou, por falta de condições da parturiente, uma vez que nem todas as terapias são recomendadas em todas as fases do trabalho de parto, como mencionado em estudo de revisão sobre o tema ${ }^{24}$

O uso da hidromassagem, nesse estudo, teve o percentual mais baixo $(8,3 \%)$, o que pode ser justificado pelo problema de restrição hídrica que a cidade vivenciava na época.

A atuação de enfermeiras obstetras/obstetrizes nos Centros de Parto Normal contribuem para qualificar e humanizar a assistência obstétrica, oferecem às parturientes maior autonomia e estão mais de acordo com as recomendações da Organização Mundial de Saúde $(\mathrm{OMS})^{16,22}$

As Terapias não farmacológicas são consideradas tecnologias leves-duras ${ }^{23}$ de fácil aplicação e poderiam ser utilizadas com maior frequência no alívio da dor e conforto das parturientes.

Entretanto, as publicações sugerem que as terapias não farmacológicas de alívio da dor ainda, são utilizadas de maneira insipiente e precisam ser estimuladas $^{5,16,17,18}$

\section{CONCLUSÃO}

As enfermeiras obstetras do Centro de Parto Normal (CPN) analisado tem utilizado algumas terapias não farmacológicas com maior frequência que outras. As mais utilizadas foram a deambulação, o banho de aspersão e o balanço pélvico. Recomenda-se que mais estudos sejam realizados para avaliar se outros CPNs têm sido mais efetivos na utilização dessas terapias. 


\section{REFERÊNCIAS}

1. Brasil. Ministério da Saúde. Parto, aborto e puerpério: assistência humanizada à mulher. Brasília. Área técnica da mulher; 2003; 11-26.

2. Nilsen E, Sabatino H, Lopes MHBM. Dor e comportamento de mulheres durante o trabalho de parto e parto em diferentes posições. Rev Esc Enferm USP [online]. 2011 [acesso em 2018 maio 10]; 45(3):557-65. Disponível em: http://www.scielo.br/ $\mathrm{pdf} / \mathrm{reeusp} / \mathrm{v} 45 \mathrm{n} 3 / \mathrm{v} 45 \mathrm{n} 3 \mathrm{a} 02 . \mathrm{pdf}$

3. Barbieri M, Henrique AJ, Chors FM, Maia NL, Gabrielloni MC. Banho quente de aspersão, exercícios perineais com bola suíça e dor no trabalho de parto. Acta Paul Enferm [online]. 2013 [acesso em 2018 jan 15]; 26(5): 478-84. Disponível em: http:// www.scielo.br/pdf/ape/v26n5/a12v26n5.pdf

4. Mafetoni RR, Shimo AKK. Métodos não farmacológicos para o alívio da dor no trabalho de parto. Rev Min Enferm [online]. 2014 [acesso em 2018 maio 10];18(2):505-512. Disponível em: http://www. reme.org.br/sumario/90

5. Silva CR, Fernandes RAQ, Ferreira KASL. Terapias alternativas e complementares na assistência à parturiente: Frequência de uso e fatores associados. Revista Enferm Atual In Derm [online]. 2013 [acesso em 2018 maio 01];13(67/5): 26-32. Disponível em: http://revistaenfermagematual.com .br/ revistas/revista_5.pdf

6. Gayeski ME, Brüggemann OM. Métodos não farmacológicos para o alívio da dor no trabalho de parto: uma revisão sistemática. Texto Context Enferm [online] 2010 [acesso em 2018 maio 10];19(4):77482. Disponível em: http://www.scielo. br/pdf/ tce/ v19n4/22.pdf

7. Eler GJ, JAQUES AE. O enfermeiro e as terapias complementares para o alívio da dor. Arq Ciênc Saúde Unipar [online]. 2006 [acesso em 2018 maio 02];19(3):185-90. Disponível em: http://revistas. unipar.br/index.php/saude/ article/view/624

8. Gallo RBS, Santana LS, Marcolin AC, Ferreira CHJ, Duarte G, Quintana SM. Recursos não-farmacológicos no trabalho de parto: protocolo assistencial. Femina [online]. 2011 [acesso em 2018 maio 01]; 38(1): 44-5. Disponível em: http://files.bvs.br/ upload/ S/0100-7254/2011/v39n1/a2404.pdf
9. Silva DAO, Ramos MG, Jordão VRV, Silva RAR, Carvalho JBL, Costa MMN. Uso de métodos não farmacológicos para o alívio da dor durante o trabalho de parto normal: revisão integrativa. Rev enferm UFPE [on line]., Recife,2013; 7(esp):4161-70. Disponível em: https://periodicos.ufpe.br/revistas/ revistaenfermagem/article/view/11645/13744 .pdf

10. Mamede FV, Mamede MV, Dotto LMG. Reflexões sobre deambulação e posição materna no trabalho de parto e parto. Esc Anna Nery Enferm [online]. 2007 [acesso em 2018 maio 05];11(2):331-6. Disponível em: http://www. scielo.br/pdf/ean/v11n2/ v11n 2a23.pdf

11. Tabarro CS, Campos LB, Galli NO, Novo NF, Pereira VM. Efeito da música no trabalho de parto e no recém-nascido. Rev Esc Enferm USP [online] 2010 [acesso em 2018 maio 06];44(2):445-52. Disponível em: http://www.scielo.br/pdf/reeusp/v44n2/29

12. Nunes $\mathrm{S}$, Vargens OMC. A crioterapia como estratégia para alívio da dor no trabalho de parto: um estudo exploratório. R Enferm UERJ [online]. 2007 [acesso em 2018 maio 01];15(3):337-42. Disponível em: http://www.facenf. uerj.br/v15n3/v15 n3a03.pdf

13. Brasil. Ministério da Saúde, Secretaria de Atenção à Saúde, Departamento de ações programáticas estratégicas. Pré-natal e puerpério, atenção qualificada e humanizada. Manual técnico. Brasília. 2006. Disponivel em: http://bvsms.saude.gov.br/bvs/publicacoes/manual pre natal puerperio 3ed.pdf

14. Davim RMB, Torres GV, Dantas JC. Efetividade de estratégias não farmacológicas no alívio da dor de parturientes no trabalho de parto. Rev Esc Enferm USP [online]. 2009; [acesso em 2018 maio 03];43(2):438-45. Disponível em: http://www.scielo. $\mathrm{br} / \mathrm{pdf} / \mathrm{reeusp} / \mathrm{v} 43 \mathrm{n} 2 / \mathrm{a} 25 \mathrm{v} 43 \mathrm{n} 2 . \mathrm{pdf}$

15. Mamede FV, Almeida AM, Nakano AMS, Gomes FA, Panobianco MS. O efeito da deambulação na duração da fase ativa do trabalho de parto. Esc Anna Nery R Enferm. 2007 [acesso em 2018 maio 10];11(3):466-71. Disponível em: http://www.scielo. br/pdf/ean/v11n3/v11n3a11

16. Lobo SF, Oliveira SMJV, Schneck CA, Silva FMB Bonadio IC, Riesco MLG. Resultados maternos e neonatais em Centro de Parto Normal peri-hospitalar na cidade de São Paulo-Brasil. Rev Esc Enferm USP [online]. 2010 [acesso em 2018 maio 01];44(3):812-8. Disponível em: http://www.scielo. br/pdf/ reeusp/v44n3/37.pdf 
17. Silva FMB, Paixão TCR, Oliveira SMJV, Leite JS, Riesco MLG, Osava RH. Assistência em um centro de parto segundo as recomendações da Organização Mundial da Saúde. Rev. esc. enferm. USP [Internet]. 2013 Out [citado 2018 Maio 29]; 47(5): 1031-1038. Disponível em: http://www.scielo.br/pdf/ reeusp/v47n5/pt_0080-6234-reeusp-47-05-1031.pdf

18. Silva TC, Bisognin P, Prates LA, Bortoli CFC, Oliveira G, Ressel LB. Práticas de atenção ao parto e nascimento: uma revisão integrativa. Revista de Enfermagem do Centro-Oeste Mineiro [online]. 2017 [acesso em 2018 maio 06];7:e1294. Disponível em: http://www.seer.ufsj.edu.br/index.php/recom/ article/view/1294/1314

19. Mamede FV, Almeida AM, Souza L, Mamede MV. A dor durante o trabalho de parto: o efeito da deambulação. Rev Latino-am Enfermagem [online]. 2007 [acesso em 2018 maio 01];15(6):1157-62. Disponível em: http://www.scielo. br/pdf/rlae/v15n6/ pt 15.pdf

20. Rodrigues PC, Silva SA, Barbosa TF, Silvia CR. Uso e conhecimento das terapias alternativas complementares durante o trabalho de parto, por gestantes em um município Paulista. REENVAP [online]. 2012 [acesso 2018 maio 01];(3):65-85. Disponível em: http://fatea.br/seer3/index.php/ REENVAP /article/view/98
21. Almeida JM, Acosta LG, Pinhal MG. Conhecimento das puérperas com relação aos métodos não farmacológicos de alívio da dor do parto. Rev Min Enferm [online]. 2015 [acesso em 2018 maio 10];19(3):711-17. Disponível em: http://www.reme. org.br/artigo/ detalhes/1034

22. Pereira ALF, Lima TRL, Shoeter MS, Gouveia MSF, Nascimento SD. Resultados maternos e neonatais da assistência em casa de parto no município do Rio de Janeiro. Esc Anna Nery [online]. 2013 [acesso em 2018 maio 06];17(1):17-23. Disponível em: http://www.scielo.br/pdf/ean/v17n1 /03.pdf

23. Silva EF, Strapasson MR, Fischer ACS. Métodos não farmacológicos de alívio da dor durante trabaIho de parto e parto. R Enferm UFSM [online]. 2011 [acesso em 2018 maio 06];1(2):261-271. Disponível em: https://periodicos.ufsm.br/reufsm/article/ view/2526/1640

24. Gayeski ME, Brüggemann OM. Métodos não farmacológicos para alívio da dor no trabalho de parto: uma revisão sistemática. Texto Contexto Enferm [online]. 2010 [acesso em 2018 maio 01]; 19(4):77482. Disponível em: http://www.scielo.br/pdf/tce/ v19n4/22.pdf 